\title{
PEMBANGKIT GRUP PERSAMAAN SCHRODINGER
}

\author{
Aloysius Joakim Fernandez \\ Universitas Katolik Widya Mandira \\ fndz1586@gmail.com
}

\begin{abstract}
In this article contains the Group's concepts in mathematical analysis namely self-adjoint property and Stone Theorem. This theorem talks about generator infinitesimal of Group. Moreover, this article will discuss the concepts of Group in Schrodinger equation.
\end{abstract}

Keyword: group, self-adjoint, generator, dissipative, Schrodinger equation.

ABSTRAK. Artikel ini memuat konsep-konsep Grup dalam matematika analisi, yakni sifat self-adjoint dan Teorema Stone. Teorema Stone berbicara tentang prmbangkit infinitesimal dari Grup. Lebih jauh bahwa artikel ini berbicara tenantang konsep-konsep Grup dalam Persamaan Schrodinger.

Kata Kunci: grup, self-adjoint, pembangkit, dissipatif, persamaan Schrodinger

\section{PENDAHULUAN}

Perkembangan ilmu matematika analisis semakin terus berkembang dengan pesat. Salah satu konsep yang belakangan ini berkembang adalah konsep semigrup. Suatu persoalan persamaan diferensial dapat dibentuk ke dalam masalah Cauchy abstrak. Semigrup didefinisikan sebagai sebuah operator eksponensial, untuk $t \geq 0$. Dalam perkembangannya, semigrup berkembang menjadi konsep grup, yang didefinisikan dengan suatu operator eksponensial, namun dipenuhi untuk $-\infty<t<\infty$.

Tujuan penulisan ini adalah untuk menelaah konsep-konsep dari grup, sifat-sifat, dan juga Teorema Stone. Selanjutnya, konsep-konsep yang ada pada grup kemudian diaplikasikan pada Persamaan Schrodinger. Persamaan ini dibentuk ke dalam masalah Cauchy abstrak, kemudian dikaji tentang pembangkit infinitesimalnya. 


\section{GRUP DAN PERSAMAAN SCHRODINGER}

\subsection{Grup}

\section{Definisi 2.1.}

Sebuah keluarga $(T(t))_{t \in \mathbb{R}}$ dari operator linear terbatas pada ruang Hilbert $X$ disebut grup kontinu kuat (grup $C_{0}$ ) jika sifat-sifat berikut berlaku :
a. $T(0)=I$.
b. $T(t+s)=T(t) T(s)$ untuk $-\infty<t, s<\infty$.
c. $\lim _{t \rightarrow 0} T(t) x=x$ untuk $x \in X$.

\section{Definsi 2.2.}

Misalkan $(T(t))_{t \in \mathbb{R}}$ merupakan grup kontinu kuat pada ruang Hilbert $X$ dan misalkan $D(G)$ merupakan subruang dari $X$ yang didefinisikan sebagai berikut

$$
D(G):=\left\{x \in X: \lim _{t \rightarrow 0} \frac{T(t) x-x}{t} \text { ada }\right\} .
$$

Untuk setiap $x \in D(G)$, didefinisikan

$$
G x:=\lim _{t \rightarrow 0} \frac{T(t) x-x}{t} .
$$

Operator $G: D(G) \subseteq X \rightarrow X$ disebut pembangkit infinitesimal dari grup kontinu $\operatorname{kuat}(T(t))_{t \in \mathbb{R}}$.

Diberikan grup kontinu kuat $(T(t))_{t \in \mathbb{R}}$ dengan pembangkit $(G, D(G))$. Kemudian definisikan $T_{+}(t):=T(t)$ dan $T_{-}(t):=T(-t)$ untuk $t \geq 0$, maka $\left(T_{+}(t)\right)_{t \geq 0}$ dan $\left(T_{-}(t)\right)_{t \geq 0}$ merupakan semigrup kontinu kuat dengan masingmasing pembangkitnya secara berurutan adalah $G$ dan $-G$. Oleh karena itu, jika $G$ pembangkit dari grup $C_{0}$, maka $G$ dan $-G$ merupakan pembangkit dari semigrup kontinu kuat. Operator $G$ merupakan pembangkit dari grup kontinu kuat $T(t)$ yang didefinisikan sebagai berikut

$$
T(t):=\left\{\begin{array}{ll}
T_{+}(t) & t \geq 0 \\
T_{-}(t) & t \leq 0
\end{array} .\right.
$$

Misalkan $X$ ruang Banach dan $X^{\prime}$ merupakan dualnya. Untuk setiap $x \in X$, definisikan himpunan dualitas $F(x) \subseteq X^{\prime}$ sebagai berikut : 


$$
F(x)=\left\{x^{\prime}: x^{\prime} \in X^{\prime} \text { dan }\left\langle x^{\prime}, x\right\rangle=\|x\|^{2}=\left\|x^{\prime}\right\|^{2}\right\} .
$$

\section{Definisi 2.3.}

Suatu operator linear $G: D(G) \subseteq X \rightarrow X$ dissipatif jika untuk setiap $x \in D(G)$ terdapat $x^{\prime} \in F(x)$ sedemikian sehingga

$$
\operatorname{Re}\left\langle G x, x^{\prime}\right\rangle \leq 0
$$

\section{Teorema 2.4.}

Suatu operator linear $G: D(G) \subseteq X \rightarrow X$ dissipatif jika dan hanya jika untuk setiap $x \in D(G)$ dan $\lambda>0$ berlaku

$$
\lambda\|x\| \leq\|(\lambda I-G) x\| .
$$

\section{Definisi 2.5.}

Misalkan $H$ ruang Hilbert dengan hasil kali dalam $\langle$, $\rangle$. Operator $G: D(G) \subseteq X \rightarrow$ $X$ dikatakan :
a. $\quad$ Self-adjoint jika $G=G^{*}$
b. Skew-adjoint jika $G=-G^{*}$
c. Symetric jika $\langle G x, y\rangle=\langle x, G y\rangle$ untuk setiap $x, y \in D(G)$
d. Skew-symetric jika $\langle G x, y\rangle=-\langle x, G y\rangle$ untuk setiap $x, y \in D(G)$.

\section{Teorema 2.6}

Misalkan $(G, D(G))$ operator rapat pada ruang Hilbert $H$, maka $G$ membangkitkan grup uniter $(T(t))_{t \in \mathbb{R}}$ pada $H$ jika dan hanya jika $i G$ self-adjoint, yakni $(i G)^{*}=$ $i G$.

\section{Bukti}

Asumsikan $(G, D(G))$ operator rapat yang membangkitkan suatu grup uniter $(T(t))_{t \in \mathbb{R}}$, maka berlaku, untuk setiap $x \in D(G)$,

$$
\begin{aligned}
-G x & =\lim _{t \searrow 0} \frac{1}{t}(T(-t) x-x) \\
& =\lim _{t \searrow 0} \frac{1}{t}\left((T(t))^{-1} x-x\right)
\end{aligned}
$$




$$
\begin{aligned}
& =\lim _{t \unlhd 0} \frac{1}{t}\left((T(t))^{*} x-x\right) \\
& =G^{*} x .
\end{aligned}
$$

Diperoleh bahwa $G^{*}=-G$. Sebagai akibatnya, $(i G)^{*}=-i G^{*}=i G$. Jadi $i G$ merupakan operator self-adjoint.

Sebaliknya, asumsikan operator pada $i G$ self-adjoint, maka $G^{*}=-G$. Akibatnya,

$$
\langle G x, x\rangle=\left\langle x, G^{*} x\right\rangle=-\langle x, G x\rangle=-\langle\bar{G} x, x\rangle,
$$

untuk setiap $x \in D(G)=D\left(G^{*}\right)$ dan $\langle G x, x\rangle \in i \mathbb{R}$, sehingga $\operatorname{Re}\langle G x, x\rangle=0$ dan operator $G$ dissipatif. Karena $G^{*}=-G$, maka $\operatorname{Re}\left\langle G^{*} x, x\right\rangle=0$ untuk $x \in D(G)$. Akibatnya, operator $G^{*}$ dissipatif. Diperoleh bahwa operator $G$ dan $G^{*}=-G$ merupakan pembangkit infinitesimal dari semigrup $C_{0}$. Jika semigrup kontinu kuat $T_{+}(t)$ dan $T_{-}(t)$ dibangkitkan oleh operator $G$ dan $G^{*}$, yang didefinisikan oleh

$$
T(t):= \begin{cases}T_{+}(t) & t \geq 0 \\ T_{-}(t) & t \leq 0\end{cases}
$$

maka $(T(t))_{t \in \mathbb{R}}$ grup uniter pada $H$.

\section{Teorema 2.7.}

Misalkan $(T(t))$ semigrup $C_{0}$ dari operator terbatas. Jika untuk setiap $t>0,(T(t))^{-1}$ ada dan merupakan suatu operator terbatas maka $S(t)=(T(t))^{-1}$ merupakan semigrup $C_{0}$ operator terbatas yang memiliki pembangkit infinitesimal $-G$. Lebih jauh

$$
U(t)=\left\{\begin{array}{cc}
T(t) & t \geq 0 \\
T(t)^{-1} & t \leq 0
\end{array}\right.
$$

sehingga $U(t)$ merupakan grup $C_{0}$ operator terbatas.

\section{Bukti}

Pertama-tama akan ditunjukkan bahwa $S(t)$ memenuhi sifat-sifat dari semigrup, yaitu

$$
\begin{gathered}
S(0)=T(0)^{-1}=I, \\
S(t+s)=T(t+s)^{-1}=(T(t) T(s))^{-1}=T(t)^{-1} T(s)^{-1}=S(t) S(s) .
\end{gathered}
$$


Selanjutnya akan ditunjukkan bahwa $S(t)$ kontinu kuat. Untuk $s>0$, daerah hasil $T(s)$ berada di dalam $X$. Misalkan $x \in X$ dan $s>1$. Terdapat $y \in X$ sedemikian sehingga $T(s) y=x$. Untuk $t<1$, diperoleh bahwa

$$
\begin{aligned}
\left\|T(t)^{-1} x-x\right\| & =\left\|T(t)^{-1} T(s) y-T(s) y\right\| \\
& =\left\|T(t)^{-1} T(s) T(t) T(t)^{-1} y-T(s) y\right\| \\
& =\left\|T(t)^{-1} T(t) T(s) T(-t) y-T(s) y\right\| \\
& =\left\|T(t)^{-1} T(t) T(s-t) y-T(s) y\right\| \\
& =\|T(s-t) y-T(s) y\| .
\end{aligned}
$$

Untuk $t \searrow 0$, diperoleh bahwa

$$
\|T(s-t) y-T(s) y\| \rightarrow 0 .
$$

Oleh karena itu, $S(t)$ kontinu kuat. Untuk $x \in D(G)$ diperoleh bahwa

$$
\begin{aligned}
\lim _{t \searrow 0} \frac{T(t)^{-1} x-x}{t} & =\lim _{t \searrow 0} T(t) \frac{T(t)^{-1} x-x}{t} \\
& =\lim _{t \unlhd 0} \frac{x-T(t) x}{t} \\
& =-G x .
\end{aligned}
$$

Sehingga operator $-G$ merupakan pembangkit infinitesimal.

\section{Teorema 2.8 (Pertubasi).}

Misalkan $A$ dan $B$ adalah operator linear pada $H$ sehingga $D(B) \subset D(A)$ dan $A+t B$ adalah dissipatif untuk $0 \leq t \leq 1$ jika

$$
\|B x\| \leq \alpha\|A x\|+\beta\|x\| \text { untuk } x \in D(A)
$$

di mana $0 \leq \alpha<1, \beta \geq 0$ dan untuk suatu $t_{0} \epsilon[0,1], A+t_{0} B$ adalah m-dissipatif, maka $A+t B$ adalah m-dissipatif untuk semua $t \epsilon[0,1]$.

\section{Teorema 2.9 (Stone).}

Operator $A: D(A) \subseteq H \rightarrow H$ pembangkit infinitesimal Grup- $C_{0}$ dari operator uniter pada Ruang Hilbert $H$ jika dan hanya jika $i A$ adalah self-adjoint.

\subsection{Persamaan Schrodinger}

Pandang persamaan Schordinger 


$$
\frac{1}{i} \frac{\partial u}{\partial t}=\Delta u-V u
$$

di mana $V$ merupakan Potensial. Persamaan ini dapat ditulis ke dalam bentuk masalah Cauchy Abstrak

$$
\begin{aligned}
\frac{1}{i} \frac{\partial u}{\partial t} & =\Delta u-V u \\
\frac{\partial u}{\partial t} & =i(\Delta u-V u) \\
\frac{\partial u}{\partial t} & =i \Delta u-i V u \\
\frac{\partial u}{\partial t} & =(i \Delta-i V) u \\
\frac{\partial u}{\partial t} & =\left(A_{0}-i V\right) u
\end{aligned}
$$

di mana $i \Delta=A_{0}$. Dengan demikian, dapat dibentuk masalah Cauchy abstrak sebagai berikut

$$
\begin{aligned}
\frac{\partial}{\partial t} u(t, x) & =\left(A_{0}-i V\right) u(t, x) \\
u(0, x) & =f(x) .
\end{aligned}
$$

Akan diperlihatkan bahwa operator $A_{0}-i V$ membangkitkan suatu grup uniter $e^{\left(A_{0}-i V\right) t}$ dalam ruang Hilbert $H=L^{2}\left(\mathbb{R}^{n}\right)$ dengan $i \Delta=A_{0}$, sehingga solusi umum dari masalah Cauchy abstrak tersebut adalah

$$
u(t, x)=f(x) e^{\left(A_{0}-i V\right) t} .
$$

\section{Definisi 2.10.}

Misalkan $D\left(A_{0}\right)=H^{2}\left(\mathbb{R}^{n}\right)$ di mana ruang $H^{2}\left(\mathbb{R}^{n}\right)$ didefinisikan sebagai

$$
H^{2}\left(\mathbb{R}^{n}\right)=\left\{f \in L^{2}\left(\mathbb{R}^{n}\right): \int_{\left(\mathbb{R}^{n}\right)}\left(1+|\xi|^{2}\right)^{2}|\hat{f}(\xi)|^{2} d \xi<\infty\right\}
$$

dengan hasil kali dalam $\langle f, g\rangle=\int_{\left.\mathbb{R}^{n}\right)}\left(1+|\xi|^{2}\right)^{k} \hat{f}(\xi) \overline{\hat{g}(\xi)} d \xi$. Untuk $u \epsilon D\left(A_{0}\right)$, misalkan $i \Delta u=A_{0} u$. 


\section{Lemma 2.11.}

Operator $i A_{0}$ self-adjoint di $L^{2}\left(\mathbb{R}^{n}\right)$.

\section{Bukti}

Perhatikan

$$
\begin{aligned}
\left\langle i A_{0} u, v\right\rangle_{0} & =\langle i i \Delta u, v\rangle_{0} \\
& =\langle-\Delta u, v\rangle_{0} \\
& =-\int_{\mathbb{R}^{n}} \Delta u \cdot \bar{v} d x \\
& =-\int_{\mathbb{R}^{n}} u \cdot \overline{\Delta v} d x \\
& =\langle u,-\Delta v\rangle_{0} \\
& =\langle u, i i \Delta v\rangle_{0} \\
& =\left\langle u, i A_{0} v\right\rangle_{0} .
\end{aligned}
$$

Selanjutnya, akan ditunjukkan bahwa operator $A_{0}-i V$ merupakan pembangkit dari grup uniter pada $L^{2}\left(\mathbb{R}^{n}\right)$. Namun demikian, terlebih dahulu akan ditunjukkan juga bahwa operator ini memenuhi sifat-sifat self adjoint dan m-dissipatif. Misalkan $D\left(A_{0}\right)=H^{2}\left(\mathbb{R}^{n}\right)$.

1. Untuk $u \in D\left(A_{0}\right)$, misalkan

$$
A_{0} u=i \Delta u \text { atau } i A_{0} u=-\Delta u .
$$

Akan ditunjukkan bahwa $i A_{0}$ memenuhi sifat self-adjoint di $L^{2}\left(\mathbb{R}^{n}\right)$.

Misalkan $\left(i A_{0}\right)^{*}$ adalah operator adjoint dari $i A_{0}$, yaitu

$$
\left\langle i A_{0} u, v\right\rangle=\left\langle u,\left(i A_{0}\right)^{*} v\right\rangle
$$

Selanjutnya, perhatikan bahwa

$$
\begin{aligned}
\langle-\Delta u, v\rangle & =-\int_{\mathbb{R}^{n}} \Delta u \cdot \bar{v} d x \\
& =-\int_{\mathbb{R}^{n}} u \cdot \overline{\Delta v} d x \\
& =\langle u,-\Delta v\rangle .
\end{aligned}
$$


Dapat disimpulkan bahwa $\left\langle i A_{0} u, v\right\rangle=\left\langle u, i A_{0} v\right\rangle$ dan $i A_{0}=\left(i A_{0}\right)^{*}$ self adjoint

2. Akan ditunjukkan bahwa operator $i A_{0}+V=i\left(A_{0}-i V\right)$ self adjoint, yaitu

$$
\begin{aligned}
\left\langle\left(i A_{0}+V\right) u, v\right\rangle & =\left\langle i A_{0} u, v\right\rangle+\langle V u, v\rangle \\
& =\left\langle u, i A_{0} v\right\rangle+\langle u, V v\rangle \\
& =\left\langle u,\left(i A_{0}+V\right) v\right\rangle .
\end{aligned}
$$

3. Akan ditunjukkan bahwa operator $\left(A_{0}-i V\right)$ bersifat m-dissipatif. Karena $i A_{0}+V$ self-adjoint maka

$$
\begin{gathered}
\left(i\left(A_{0}-i V\right)\right)^{*}=i\left(A_{0}-i V\right) \\
-i\left(A_{0}-i V\right)^{*}=i\left(A_{0}-i V\right) \\
\left(A_{0}-i V\right)^{*}=-\left(A_{0}-i V\right) .
\end{gathered}
$$

Perhatikan

$$
\begin{aligned}
\left\langle\left(A_{0}-i V\right) u, u\right\rangle & =\left\langle u,\left(A_{0}-i V\right)^{*} u\right\rangle \\
& =\left\langle u,-\left(A_{0}-i V\right) u\right\rangle \\
& =-\left\langle u,\left(A_{0}-i V\right) u\right\rangle \\
& =-\overline{\left\langle\left(A_{0}-\imath V\right) u, u\right\rangle},
\end{aligned}
$$

sehingga diperoleh bahwa $\operatorname{Re}\left\langle\left(A_{0}-i V\right) u, u\right\rangle=0$.

4. Akan ditunjukkan bahwa $A_{0}$ m-dissipatif. Karena $i A_{0}$ self-adjoint, maka $A_{0}{ }^{*}=-A_{0}$, sehingga

$$
\begin{gathered}
\left\langle A_{0} u, u\right\rangle=\left\langle u, A_{0}{ }^{*} u\right\rangle \\
=-\left\langle u, A_{0} u\right\rangle \\
=-\overline{\left\langle A_{0} u, u\right\rangle .}
\end{gathered}
$$

Akibatnya, diperoleh bahwa $\operatorname{Re}\left\langle A_{0} u, u\right\rangle=0$.

\section{Akibat 2.12.}

Operator $A_{0}$ adalah pembangkit infinitesimal dari grup operator uniter pada $L^{2}\left(\mathbb{R}^{n}\right)$

Definisikan sebuah operator $V$ di $L^{2}\left(\mathbb{R}^{n}\right)$ dengan

$$
D(V)=\left\{u: u \in L^{2}\left(\mathbb{R}^{n}\right), V \cdot u \in L^{2}\left(\mathbb{R}^{n}\right)\right\}
$$


dan untuk $u \in D(V), V u=V(x) u(x)$

\section{Lemma 2.13.}

Misalkan $V(x) \epsilon L^{p}\left(\mathbb{R}^{n}\right)$. Jika $p>\frac{n}{2}$ dan $p \geq 2$ maka untuk setiap $\varepsilon>0$ terdapat konstanta $C(\varepsilon)$ sehingga

$$
\|V u\| \leq \varepsilon\|\Delta u\|+C(\varepsilon)\|u\| \text { untuk } u \epsilon H^{2}\left(\mathbb{R}^{n}\right)
$$

di mana norm $\|$.$\| merupakan norm L^{2}$ di $\mathbb{R}^{n}$.

\section{Bukti}

Jika $u \in H^{2}\left(\mathbb{R}^{n}\right)$ maka dengan transformasi Fourier diperoleh bahwa $\left(1+|\xi|^{2}\right) \hat{u}(\xi) \epsilon L^{2}\left(\mathbb{R}^{n}\right)$ dan karena $p>\frac{n}{2}$, maka $\left(1+|\xi|^{2}\right)^{-1} \epsilon L^{p}\left(\mathbb{R}^{n}\right)$. Dengan menggunakan ketaksamaan Holder, identitas Parseval, dan untuk $q=\frac{2 p}{2+p}, \frac{1}{q}=\frac{1}{p}+\frac{1}{2}$, diperoleh bahwa

$$
\begin{aligned}
\|\hat{u}\|_{q} & =\left(\int_{\mathbb{R}^{n}}|\hat{u}(\xi)|^{q} d \xi\right)^{\frac{1}{q}} \\
& =\left(\int_{\mathbb{R}^{n}}\left(1+|\xi|^{2}\right)^{-q}\left(1+|\xi|^{2}\right)^{q}|\hat{u}(\xi)|^{q} d \xi\right)^{\frac{1}{q}} \\
& \leq\left(\int_{\mathbb{R}^{n}}\left(1+|\xi|^{2}\right)^{-p} d \xi\right)^{\frac{1}{p}}\left(\int_{\mathbb{R}^{n}}\left(1+|\xi|^{2}\right)^{2}|\hat{u}(\xi)|^{2} d \xi\right)^{\frac{1}{2}} \\
& \leq C_{p}(\|\Delta u\|+\|u\|) .
\end{aligned}
$$

Karena $p \geq 2,1 \leq q \leq 2$ dan berdasarkan pertidaksamaan Hausdorff-Young, diperoleh bahwa

$$
\|u\|_{r} \leq\|\hat{u}\|_{q} \text { di mana } \frac{1}{r}+\frac{1}{q}=1,
$$

sehingga

$$
\begin{gathered}
\|u\|_{r} \leq\|\hat{u}\|_{q} \leq C_{p}(\|\Delta u\|+\|u\|) \\
\|u\|_{r} \leq C_{p}(\|\Delta u\|+\|u\|) .
\end{gathered}
$$


Dengan mengganti fungsi $u(x)$ dengan $u(\rho x), \rho>0$ dan memilih sebuah $\rho$ yang tepat, dapat dibuat koefisien dari $\|\Delta u\|$ sekecil seperti yang diharapkan. Diberikan $\varepsilon>0$, sehingga diperoleh bahwa

$$
\|u\|_{r}\|V\|_{p} \leq \varepsilon\|\Delta u\|+C(\varepsilon)\|u\| .
$$

Dengan menggunakan ketaksamaan Holder, diperoleh bahwa

$$
\|V u\|^{2}=\int_{\mathbb{R}^{n}} V^{2} u^{2} d x \leq\left(\int_{\mathbb{R}^{n}}|V|^{p} d x\right)^{\frac{2}{p}}\left(\int_{\mathbb{R}^{n}}|u|^{r} d x\right)^{\frac{2}{r}},
$$

sehingga

$$
\begin{gathered}
\|V u\|^{2} \leq\|V\|_{p}{ }^{2}\|u\|_{r}{ }^{2} \\
\|V u\| \leq\|V\|_{p}\|u\|_{r} \leq \varepsilon\|\Delta u\|+C(\varepsilon)\|u\| .
\end{gathered}
$$

\section{Teorema 2.14 .}

Misalkan $V(x) \epsilon L^{p}\left(\mathbb{R}^{n}\right)$. Jika $p>\frac{n}{2}, p \geq 2$ maka $A_{0}-i V$ adalah pembangkit infinitesimal dari grup operator uniter pada $L^{2}\left(\mathbb{R}^{n}\right)$.

\section{Bukti}

Telah ditunjukkan bahwa operator $i A_{0}$ self adjoint dan $A_{0}$ m-dissipatif. Karena $V$ adalah operator real dan $V$ simetris, maka $i A_{0}+V$ operator simetris. Untuk membuktikan bahwa bahwa $i A_{0}+V$ self-adjoint maka perlu ditunjukkan range dari $I \pm\left(A_{0}-i V\right)$ adalah $L^{2}\left(\mathbb{R}^{n}\right)$. Berdasarkan fakta bahwa $\pm\left(A_{0}-i V\right)$ adalah $m$-dissipatif yang diperoleh dari sifat ke $m$-dissipatif $\pm A_{0}$,

$$
\|V u\| \leq \varepsilon\left\|A_{0} u\right\|+C(\varepsilon)\|u\| \text { untuk } u \in D\left(A_{0}\right)
$$

dan Teorema Pertubasi yang mengakibatkan $A_{0}-i V$ self-adjoint serta berdasarkan Teorema Stone, maka $A_{0}-i V$ adalah pembangkit infinitesimal dari grup operator uniter pada $L^{2}\left(\mathbb{R}^{n}\right)$.

\section{KESIMPULAN}

Persamaan Schrodinger ini dapat diselesaikan dengan menggunakan konsep Grup. Persamaan tersebut diubah ke dalam bentuk masalah Cauchy abstrak 


$$
\begin{aligned}
\frac{\partial}{\partial t} u(t, x) & =\left(A_{0}-i V\right) u(t, x) \\
u(0, x) & =f(x) .
\end{aligned}
$$

pada $L^{2}\left(\mathbb{R}^{n}\right)$. Selanjutnya, berdasarkan Teorema Stone, operator $A_{o}-i V$ merupakan pembangkit dari Grup uniter $e^{\left(A_{0}-i V\right) t}$ karena operator tersebut memenuhi sifat self-adjoint.

\section{DAFTAR PUSTAKA}

Pazy. A., Semigroups of Linear Operators and Application to Partial Differential Equation, Applied Mathematical Sciences, Vol 44, Springer,1983.

I.V. Joan., $C_{0}$-Semigroups and Applications, Springer, 2003.

Goldstein, J., E., Semigroups of Linear Operators and Application, Oxford University Press, 1985.

Kreyszig, E., Introductory Functional Analysis with Application, John Wiley \& Sonc, Inc., 1978. 
